\title{
Utilização de Ambientes Virtualizados para Ensino de Servidores de Redes de Computadores
}

\author{
Léo M. Lopes ${ }^{1}$, Daiany F. Lara ${ }^{1}$, Franciano Antunes', Cláudia Alves Perez ${ }^{1}$ \\ ${ }^{1}$ Universidade do Estado de Mato Grosso - (UNEMAT) - Colíder - MT - Brasil \\ \{leoneto, dflara,franciano\}@unemat.br, prof.contabeis@gmail.com
}

\begin{abstract}
The practical activities of the discipline of computer networks are essential for the formation of a competent professional, but are not always possible to perform tests that need to change the settings of machines in a lab. This paper presents a study of use of virtual environments for teaching networking and server configuration. Thus, we performed experiments, which could be tested the installation and configuration of different network services, using virtual machines as a means to simulate the client / server relationship, thus not requiring changes in the structure of the laboratory.

Resumo: As atividades práticas da disciplina de redes de computadores são fundamentais para a formação de um profissional competente, mas nem sempre é possivel realizar testes que necessitem de alterar as configurações das máquinas de um laboratório. Este trabalho traz um estudo de utilização de ambientes virtualizados para o ensino de redes e configuração de servidores. Assim, foram realizados experimentos práticos, onde puderam ser testadas a instalação e configuração de diferentes serviços de rede, utilizando como recurso máquinas virtuais simulando o relacionamento cliente/servidor, dessa maneira não necessitando de modificações na estrutura do laboratório.
\end{abstract}

\section{Introdução}

A prática em laboratório é um componente fundamental do ensino de redes de computadores. Em experimentos mais complexos, muitas vezes torna-se necessário adequar à topologia da rede do laboratório a cada experimento e alterar as configurações do sistema operacional de cada máquina (NABHEN, 2006).

Para (NABHEN, 2006) essa característica não convencional das atividades práticas da disciplina de redes de computadores (que aparece também em algumas outras disciplinas da área de sistemas, como sistemas operacionais) exige a disponibilidade de um laboratório específico e exclusivo para as atividades dos alunos nessa disciplina. Segundo o autor nem sempre é possível ter disponível essa estrutura na maioria das instituições de ensino, mas então como fazer para aplicar os conhecimentos adquiridos na teoria sem prejudicar o funcionamento do laboratório? A resposta mais sensata pode ser a utilização de aplicativos que tornam possíveis estes testes sem prejudicar o sistema operacional da máquina, ou seja, disponibilizam um ambiente virtualizado, ou simplesmente: Máquina Virtual (Virtual Machine).

A origem da virtualização remonta nos anos 70 (CARISSIMI, 2010), quando existiam 
V Congresso Brasileiro de Informática na Educação (CBIE 2016)

Anais do XXVII Simpósio Brasileiro de Informática na Educação (SBIE 2016)

apenas algumas fabricantes de software e hardware geralmente fechados, ou seja, sistemas que só funcionavam no hardware do mesmo fabricante. Portanto se viu a necessidade de criar ambientes que pudessem emular este hardware proprietário mesmo rodando em uma máquina totalmente diferente.

Atualmente a técnica de virtualização vem ganhando destaque na Tecnologia da Informação (TI) sendo muito utilizada em servidores de redes, desta forma aproveitando melhor os recursos da máquina disponível. Porém sua utilização não se limita a este ambiente, pois as máquinas virtuais podem ser utilizadas em diversos setores das organizações, até mesmo por usuários domésticos e universidades em ambientes de testes.

Este artigo traz um estudo sobre a viabilidade da técnica de virtualização de sistemas operacionais em um ambiente de testes (laboratório) utilizado em cursos de servidores de redes. Para tanto, foram realizados 02 (dois) experimentos através de cursos sobre configuração de serviços em servidores de rede.

\section{Redes de Computadores}

Uma rede de computadores consiste de 2 ou mais computadores e outros dispositivos conectados entre si de modo a poderem compartilhar seus serviços, que podem ser: dados, impressoras, mensagens (e-mails), etc. A Internet é um amplo sistema de comunicação que conecta muitas redes de computadores. Existem várias formas e recursos (equipamentos) que podem ser interligados e compartilhados, mediante meios de acesso, protocolos e requisitos de segurança (HANSEN, 2009).

Segundo (HANSEN, 2009) existem diversas topologias de redes, mas o modelo de rede Cliente/Servidor se destaca em relação aos outros. Este e amplamente usado e constitui a base da grande utilização da rede. Ele e aplicável quando o cliente e o servidor estão ambos no mesmo edifício (por exemplo, quando um servidor de acesso à Intenet ou Intranet pertence à mesma empresa), mas também quando estão muito distantes um do outro. Por exemplo, quando uma pessoa em sua casa acessa uma pagina na World Wide Web, é empregado o mesmo modelo, com o servidor da Web remoto fazendo o papel do servidor e o computador pessoal do usuário sendo o cliente.

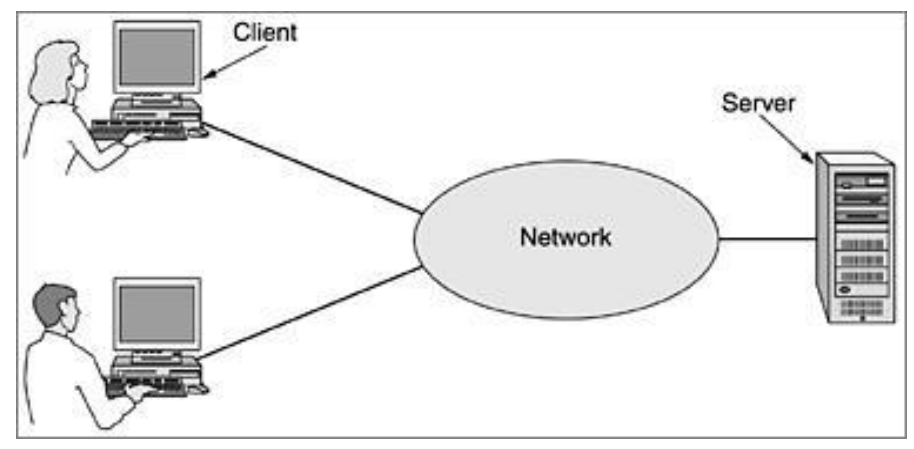

Figura 1: Rede Cliente/Servidor (TANENBAUM, 2003). 
V Congresso Brasileiro de Informática na Educação (CBIE 2016)

Anais do XXVII Simpósio Brasileiro de Informática na Educação (SBIE 2016)

Um servidor é uma máquina que fica o tempo toda ligada, sempre fazendo a mesma coisa. Existem vários tipos de servidores, como servidores Web, servidores de arquivos, servidores de impressão, acesso à Internet, etc., sendo que uma única máquina pode rodar simultaneamente vários serviços, dependendo apenas da configuração de hardware e da demanda (MORIMOTO, 2006).

Portanto o estudo de servidores de redes apresenta um importante aspecto da formação de profissionais da área de Tecnologia da Informação, pois mesmo que este profissional não trabalhe diretamente na administração desta rede é fundamental entender o seu funcionamento.

\section{Ensino de Redes de Computadores}

No modelo atual de ensino, as práticas em laboratório são cada vez mais comuns, e em particular no ensino da implementação e manutenção das próprias interligações que viabilizam as redes de computadores. As aulas práticas devem colocar o aprendiz em contato com eventos reais que ocorrem no mundo das redes, permitindo que ele vivencie experiências que lhe possibilitem passar de mero expectador como, por exemplo, um aluno que apenas assiste às aulas (ensino baseado em simples transmissão de informações), para um participante colaborador que experimenta, delimita o problema, e através da convivência com a situação real, raciocina, deduz, interage com colegas, com o material instrucional e com o professor e transforma-se de assistente a agente motriz do seu próprio aprendizado (BIANCHINI, 2008).

Para (HANSEN, 2009) um dos grandes problemas existentes no ensino de Redes de Computadores é a estrutura física para aulas práticas. Quando existem laboratórios específicos para o ensino deste conteúdo, as turmas de alunos têm que ser divididas em grupos, pois a quantidade de equipamentos é reduzida.

Segundo (NABHEN, 2009) nem sempre é possível ter a disposição uma estrutura específica; a realidade da maioria das instituições de ensino impõe o uso de laboratórios compartilhados com diversas turmas e disciplinas, o que inviabiliza a realização de experimentos mais elaborados, que exijam exclusividade no uso do laboratório. Esse problema pode ser contornado através do uso de máquinas virtuais.

\section{Máquina Virtual (Virtualização)}

Existem muitas definições de Máquina Virtual ou Virtual Machine (VM), mas basicamente é uma duplicata isolada de uma máquina real (CARISSIMI, 2010 apud POPEK e GOLDBERG, 1974), ou seja, é a execução de diversos sistemas operacionais dentro de uma mesma máquina real. Segundo (GOMES, 2007) "Virtualização refere-se a tecnologias criadas para fornecer uma camada de abstração entre sistemas de hardware de computador e o software que roda nestes sistemas" esta camada refere-se ao hypervisor ou Virtual Machine Monitor - VMM, que funciona entre o hardware hospedeiro e o Sistema Operacional virtualizado, gerenciando o uso dos recursos reais. 


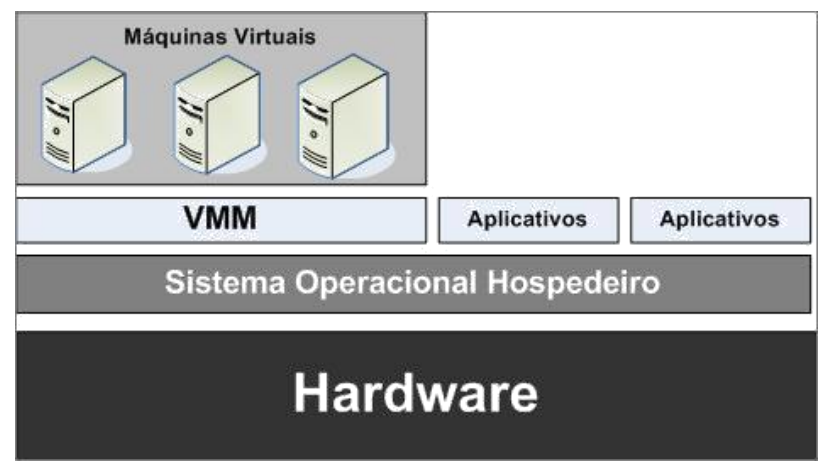

Figura 2: Estrutura virtualização

Segundo (MATTOS, 2009) em um ambiente virtualizado, temos que definir mais dois conceitos: os de Sistema Operacional Hospedeiro e o de Sistema Operacional Visitante. O primeiro, Sistema Operacional hospedeiro (Host Operating System), referese ao sistema operacional nativo da máquina na qual ocorrerá a virtualização, ou seja, este é o sistema operacional que é executado diretamente sobre o hardware físico. $\mathrm{O}$ segundo, sistema operacional visitante (Gest Operating System) refere-se ao sistema operacional que é executado sobre o hardware virtualizado, isto é, o sistema operacional que é executado na máquina virtual. Uma máquina na qual é feita a virtualização pode contar com apenas um SO hospedeiro sendo executado por vez. No entanto, podem ser executados diversos SOs visitantes simultaneamente. A virtualização pode ocorrer de dois níveis funcionais, sendo: virtualização completa e virtualização parcial (paravirtualização).

\section{Trabalhos Correlatos}

Antes de planejar execução desse trabalho, uma série de nortearam as a elaboração na metodologia adequada para que estudos relacionados objetivos esperados.

se pudesse alcançar os

Muitos estudos propões a utilização do ensino de redes com a utilização do emulador de redes de computadores NetKit (RIMONDINI, 2007), como é demonstrado em Gurgel et al (2012) onde 10 temas foram abordados utilizando esta ferramenta como recurso pedagógico, Gurgel ainda apresenta uma avaliação sobre o desempenho da metodologia nos aspectos nível de profundidade e nível de conhecimento, sobre cada um dos temas trabalhados.

Maciel (2014), apresenta a utilização de emuladores para o ensino de redes de computadores e realize a avaliação e comparação de três emuladores de redes disponíveis gratuitamente, sendo eles, o Core, o Netkit e o Mininet. Araújo (2012), também demonstra a criação de laboratórios virtuais com fins didáticos utilizando os emuladores de redes NetKit e NetGui.

Nabhen e Maziero (2006), semelhante a este estudo, apresenta a aplicação do uso de máquinas virtuais para o ensino de redes de computadores, com o objetivo de superar a falta de laboratórios para implementações práticas, e relatam a viabilidade da utilização de máquinas virtuais, destacando o maior envolvimento dos alunos e melhor aproveitamento dos créditos destinados a aulas práticas.

Silva (2014), demonstra a utilização de máquinas virtuais como metodologia de ensino na disciplina de Gestão Sistemas Operacionais, Silva ainda realiza uma avaliação da experiência sob a perspectiva dos alunos. 
V Congresso Brasileiro de Informática na Educação (CBIE 2016)

Anais do XXVII Simpósio Brasileiro de Informática na Educação (SBIE 2016)

\section{Experimentos Realizados}

Dois cursos foram elaborados com o objetivo de evidenciar a viabilidade de utilização de máquinas virtuais para ensino de servidores de rede. Os cursos foram realizados em dois momentos e instituições diferentes, sendo o primeiro curso aqui denominado Experimento 1 abordou o tema "Servidor Proxy completo com Linux Ubuntu Server" e o segundo curso aqui denominado Experimento 2 com o tema "Servidor Web completo com Ubuntu Server".

Em ambos os experimentos foram utilizados o software para virtualização total VirtualBox desenvolvido pela Sun Microsystems que recentemente foi comprada pela Oracle. Este foi escolhido pelas seguintes características:

- Virtualização total (não há necessidade de modificar o SO);

- Facilidade de utilização principalmente por usuários iniciantes;

- Gratuito;

- Totalmente em português;

- Disponível para as duas plataformas (Windows e Linux).

\subsection{Experimento 1}

Foi realizado no laboratório de informática da Universidade do Estado de Mato Grosso durante a $1^{\text {a }}$ Semana de Computação, com 20 alunos do curso de Licenciatura em Computação da referida universidade.

Neste curso o objetivo foi implementar uma topologia cliente/servidor disponibilizando o acesso a Internet através de um servidor Proxy, com os seguintes serviços:

- DHCP (Dynamic Host Controll Protocol): responsável pelo endereçamento dinâmico das máquinas em uma rede;

- Squid: controla o acesso a Internet e armazena informações de páginas web acessadas pelos usuários (cache) melhorando o desempenho da rede;

- Iptables: firewall da rede, controla de tudo que entra e sai da rede;

- Bind: serviço de DNS (Domain Name Server) faz a tradução do nome para endereço IP dos sites;

\subsection{Recursos e procedimentos}

Foram utilizadas 20 máquinas do laboratório com seguinte configuração:

- Processador Dual Core 2.0 Ghz;

- 1 GB de memória RAM;

- 160 GB de HD;

- Sistema Operacional Linux Ubuntu Desktop 9.10;

- Acesso a Internet; 
V Congresso Brasileiro de Informática na Educação (CBIE 2016)

Anais do XXVII Simpósio Brasileiro de Informática na Educação (SBIE 2016)

Foi solicitado ao setor de suporte da universidade a instalação do Oracle VirtualBox 4.0 em todas as máquinas, esta foi a única alteração na máquina hospedeira.

Inicialmente os alunos conheceram as funcionalidades do VirtualBox e adicionaram as máquinas virtuais (VM) com as configurações de rede necessárias:

\section{VM Servidor:}

- Sistema Operacional Linux Ubuntu Server 9.10;

- 2 Placas de rede, sendo a primeira (eth0) em modo NAT, nesta configuração a VM tem acesso a rede local do laboratório através da máquina hospedeira utilizando o serviço Network Address Translate - NAT (Tradução de Endereço de Rede) gerenciado pelo próprio VirtualBox e a segunda placa (eth1) configurada como rede interna (nesta configuração apenas as outras VMs tem acesso);

\section{VM Cliente 1:}

- Sistema Operacional Microsoft Windows XP;

- 1 Placa de rede configurada como rede interna;

\section{VM Cliente 2:}

- Sistema Operacional Linux Ubuntu 9.10 Desktop;

- 1 Placa de rede configurada como rede interna;

Na figura 4 é mostrada a tela do VirtualBox com as 3 VM já adicionadas.

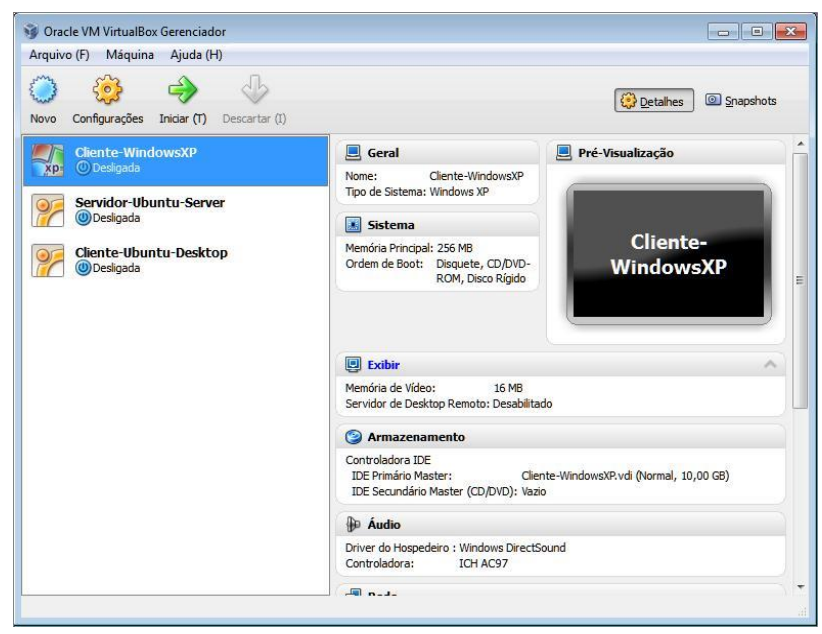

Figura 3: Janela do Virtual com as VM's utilizadas

Após a instalação e configuração de todos os pacotes (serviços) do servidor foram realizados testes de conexão entre as máquinas virtuais VM Servidor e os dois clientes, inclusive com as 3 máquinas virtuais funcionando ao mesmo tempo. 
V Congresso Brasileiro de Informática na Educação (CBIE 2016)

Anais do XXVII Simpósio Brasileiro de Informática na Educação (SBIE 2016)

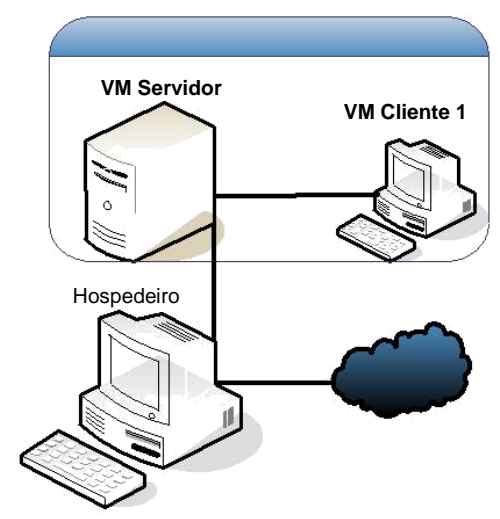

Figura 4: Experimento 1 - Teste 1

No primeiro teste do Experimento 1 a VM Cliente 1 se conectou a Internet através da VM Servidor, com os serviços de Proxy acima citados todos rodando. Por sua vez o servidor saiu para Internet através do hospedeiro.

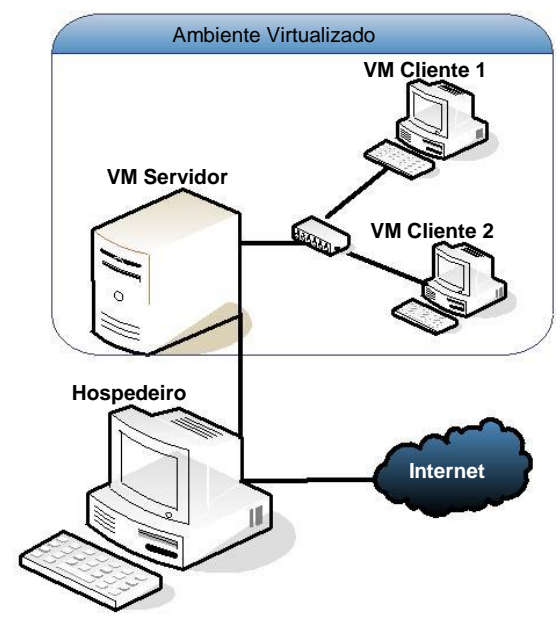

Figura 5: Experimento 1 - Teste 2

No segundo teste utilizou-se os dois clientes, conectados a um Switch virtual adicionado pelo VirtualBox, saindo para a Internet através da VM Servidor. Neste teste foi possível ainda verificar as possibilidades do controle de acesso a Internet através do serviço Squid, isolando os clientes em duas redes virtuais diferentes, inclusive com políticas de acesso a Internet específica para cada subrede através da configuração do IP ${ }^{1}$ atribuído pelo serviço DHCP. Foi implementado ainda regras de firewall com comando IpTables controlando todas as conexões entrando e saindo da rede passando pela VM Servidor.

\subsection{Experimento 2}

O segundo experimento foi denominado "Servidor Web completo com Ubuntu Server" realizado no laboratório de informática da Faculdade do Pantanal com 20 alunos do curso de Sistemas da Informação.

\footnotetext{
${ }^{1}$ Internet Protocol, número de identificação de um host na rede TCP/IP.
} 
V Congresso Brasileiro de Informática na Educação (CBIE 2016)

Anais do XXVII Simpósio Brasileiro de Informática na Educação (SBIE 2016)

Neste curso o objetivo foi configurar um servidor capaz de hospedar páginas web com os seguintes serviços:

- Apache22: responsável por atender as requisições aos arquivo do site;

- PHP53: interpretador de linguagem PHP;

- MySQL4: banco de dados;

- PHPMyAdmin5: gerenciador de banco de dados;

- SSH6: protocolo para acesso remoto e transferência de arquivos.

\subsection{Recursos e procedimentos}

Foram utilizadas 20 máquinas do laboratório de informática com a seguinte configuração:

- Processador Dual Core 2.6 Ghz;

- 2 GB de memória RAM;

- $160 \mathrm{~GB}$ de HD;

- Sistema operacional Windows 7 Starter Edition, sendo que este laboratório não possuía conexão com a Internet.

Devido este laboratório ser exclusivo do curso de Sistemas da Informação, houve a possibilidade dos próprios alunos instalarem o programa VirtualBox através de uma conta com privilégios de Administrador do Sistema.

Seguindo a mesma metodologia do Experimento 1, fez-se uma abordagem rápida das funcionalidades do VirtualBox, com a adição de 02 máquinas virtuais.

\section{VM Servidor:}

- Sistema Operacional Linux Ubuntu Server 9.10;

- 02 Placas de rede, sendo a primeira (eth0) em modo Bridge (ponte), nesta configuração a VM tem acesso direto a rede local do laboratório como se fosse uma máquina real e a segunda placa (eth1) configurada como rede interna (nesta configuração apenas as outras VMs tem acesso);

\section{VM Cliente:}

- Sistema Operacional Microsoft Windows XP;

- 01 Placa de rede configurada como rede interna;

Após a instalação e configuração de todos os pacotes (serviços) no servidor foram realizados testes de conexão entre o servidor e a VM cliente, simulando o que acontece na Internet, onde uma máquina cliente acessa uma página web hospedada em um

\footnotetext{
2 http://www.apache.org

${ }_{4}^{3} \mathrm{http}: / /$ www.php.org

${ }_{5}^{4} \mathrm{http}: / /$ www.mysql.org

${ }_{6} \mathrm{http}: / /$ www.phpmyadmin.org

6 http://www.openssh.org
} 
V Congresso Brasileiro de Informática na Educação (CBIE 2016)

Anais do XXVII Simpósio Brasileiro de Informática na Educação (SBIE 2016)

servidor remoto. Além disso, os alunos fizeram a transferência de arquivos entre as duas máquinas virtuais, com o protocolo SSH e adicionaram uma base de dados no MySQL utilizando o PHPMyAdmin conectando remotamente na VM Servidor através da VM Cliente.

\section{Análise de Resultados}

Através dos dois experimentos realizados foi possível verificar que as aulas práticas são indispensáveis para a formação de um bom profissional para atuar no ramo de redes de computadores, para tanto no modelo tradicional exige-se a disponibilidade de um laboratório exclusivo para essa disciplina, devido a necessidade de executar testes com diferentes sistemas operacionais e topologias de redes.

Porém a utilização de máquinas virtuais se mostrou muito eficaz nos testes realizados, pois atendeu todas as necessidades de configuração e instalação de sistemas operacionais que possibilitasse o objetivo do curso.

No Experimento 1, como a finalidade era a instalação e configuração de um proxy de rede, foi possível realizar diversos testes com configurações e topologias de redes deferentes, sem alterar a estrutura do laboratório.

O Experimento 2 foi fundamental o emprego da virtualização, pois no laboratório utilizado não havia conexão de rede entre os computadores, portanto impossibilitaria os testes cliente/servidor, mas com a conexão entre as máquinas virtuais foi possível atingir o objetivo.

\section{Considerações Finais}

Pretendeu-se neste trabalho estudar a viabilidade da utilização da técnica de virtualização em aulas práticas de redes de computadores, para satisfazer este objetivo optou-se em apresentar os conceitos necessários para o entendimento do tema apresentado, logo após uma descrição seqüencial dos experimentos desenvolvidos em duas oportunidades distintas através de cursos práticas de servidores de redes. Pensa-se que este trabalho alcançou seu objetivo, pois foi possível constatar que o emprego desta técnica viabiliza o desenvolvimento de testes práticos mais complexos, enriquecendo o processo de aprendizagem do aluno.

Apesar do nível de virtualização utilizado (virtualização total) consumir mais recursos da máquina hospedeira, verificou-se a possibilidade de instalação e utilização de diversas máquinas virtuais simultaneamente, garantindo o desempenho necessário para os testes. Outro fator importante foi a oportunidade dos alunos continuarem os testes desenvolvidos na aula em seus computadores pessoais, devido a facilidade migrar as máquinas virtuais, bem como as configurações do VitualBox para qualquer computador, independente do hardware, sistema operacional e topologia de rede.

Considera-se relevante para trabalhos futuros a comparação de desempenho de outros programas voltados pra virtualização, bem como a utilização das duas técnicas: virtualização total e paravirtualização. 
V Congresso Brasileiro de Informática na Educação (CBIE 2016)

Anais do XXVII Simpósio Brasileiro de Informática na Educação (SBIE 2016)

\section{Referências}

ARAÚJO, D. J. R., BOGO, M. Simulação de Ambientes de Rede para Suporte ao Ensino de Redes de Computadores: Projeto e Criação de Novos Ambientes. In: ENCONTRO DE COMPUTAÇÃO E INFORMÁTICA DO TOCANTINS, 14., 2012, Palmas. Anais... Palmas: CEULP/ULBRA, 2012. p. 33-42.

BIANCHINI, David. et al. Laboratório virtual Interativo no ensino de rede de computadores: a experiência da PUC Campinas. Campinas - SP, 2008.

CARISSIMI, A. Virtualização: da teoria a soluções. UFRGS. Porto Alegre - RS, 2010.

COELHO, Fabio de Azevedo. et al. Virtualização. Disponível em: http://www.gta.ufrj.br/grad/09_1/versao-final/virtualizacao/index.html. Acessado em fevereiro de 2015.

GOMES, Marcelo Marques. Máquinas Virtuais. UNILINHARES Linhares - SP, 2007.

GURGEL, P. H., BARBOSA, E. F. e BRANCO, K. C. "A ferramenta Netkit e a virtualização aplicada ao ensino e aprendizagem de redes de computadores". Em:

Workshop de Ensino em Informática - WEI, Anais do $32^{\circ}$ Congresso da Sociedade Brasileira de Computação, Curitiba.2012.

HANSEN, J. C. e SCHAEFFER, C. A. L. Estudo e aplicação de virtualização na criação de ambientes para ensino de redes de computadores. Universidade de Passo Fundo. Passo Fundo - RS, 2009.

KUROSE, J. F; ROSS, K. W. Redes de Computadores e a Internet: uma nova abordagem. 1. ed. São Paulo: Addison Wesley, 2003.

MACIEL, M. A. S. Avaliação de Emuladores para o Ensino de Redes de Computadores. INFOBRASIL, Fortaleza. 2014.

MATTOS, Diogo Menezes Ferrazani. Virtualização: VMWare e Xen. UFRJ. Rio de Janeiro - RJ, 2009.

MORIMOTO, C. E.. Linux, Redes e Servidores: guia prático. $2^{\text {a }}$ Ed. Atualizada. 2006. Disponível em: www.guiadohardware.net. Acessado em fevereiro de 2015.

NABHEN, C. MAZIERO. R. Experiências no uso de máquinas virtuais para o ensino de redes de computadores. In: XIV Workshop sobre Educação em Computação. Campo Grande. 2006.

TANENBAUM. Andrew S. Redes de computadores. 4. ed. Editora Campos, 2003.

RIMONDINI, M. (2007). "Emulation of Computer Networks with Netkit." http://www.netkit.org/publications/netkit-tr.pdf, Abril.

SILVA, G. Análise da Utilização de Máquinas Virtuais no Ensino da Disciplina Gestão de Sistemas Operacionais. In. III Congresso Internacional de Ciência, Tecnologia e Desenvolvimento. Taubaté. 2014. 